\title{
Déterminants de la qualité des produits végétaux
}

Marianne LE BAIL

AgroParisTech,

UMR SADapt,

Equipe qualité prod, systèmes, organisations,

Département Sciences \& Ingénierie

agronomiques, forestières, de l'eau

et de l'environnement,

AGROPARISTECH,

16 Rue Claude Bernard,

75631 Paris cedex 05

<lebail@agroparistech.fr>

Sur cette question de la qualité, je développerai et ferai valoir ici le point de vue de l'agronome. Naturellement, il n'est pas possible dans le cadre d'un exposé de passer en revue l'ensemble des déterminants de la qualité des productions végétales. Je vous ferai part plus modestement d'une réflexion qui s'élabore à I'intersection des filières et des espaces de production agricoles, une interface qui apparaît comme stratégique dans la mesure où elle concentre l'offre et que c'est là où essentiellement se font la fabrication et la production de la qualité.

J'examinerai en premier lieu les questions que pose la qualité à l'agronome et présenterai les deux postures qu'il lui est possible d'adopter face à ce nouvel objet d'étude. La première qui envisage la qualité comme un compromis entre les différentes caractéristiques des plantes, la seconde pour laquelle la qualité résulte d'un compromis entre différentes organisations. Une double approche qui devrait permettre de mieux circonscrire cette question aujourd'hui cruciale, à savoir : se dirige-t-on vers une qualité durable?

Traditionnellement, les questions agricoles pour l'agronome tournent essentiellement autour de la production, des objets et des échelles associés : I'organe et la plante, la parcelle et le peuplement végétal, la parcelle et les soles des exploitations. Tous objets d'étude qui donnent lieu à des travaux portant sur les systèmes de culture, le fonctionnement des plantes, etc.

L'association avec les économistes et la prise en compte du problème de la qualité conduisent I'agronome à élargir son champ de recherche et à porter son attention, au-delà du strict domaine de la production, sur les questions de définition (un objet de recherche en soi) et de garantie - garantie auprès du consommateur

\begin{abstract}
From the point of view of the agronomist, quality is not only a sum of characteristics of crops to be reached at the field but also a question of organization between farms and agro-food chains which collect and transform these products. To study the determinant factors of quality impose to study its definition, production and guarantee as compromise between biologic and technical components on one hand and the economic components integrating coordinations among actors on the other hand. Some of these approaches are presented by showing the transfers of scale between the plot of land, the farm and the crop supply system of agro-food chains needed to resolve questions of quality.

Key words: agro-food chains, agronomist quality, crops
\end{abstract}

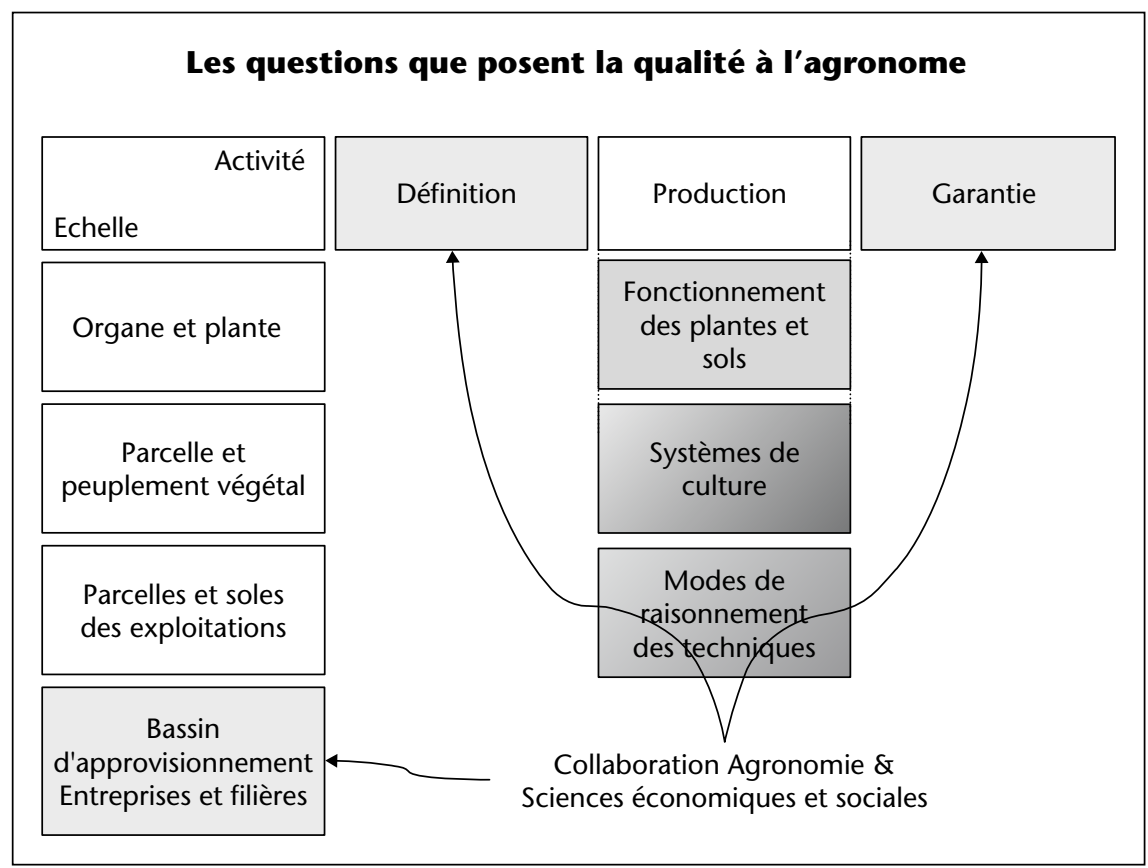

Figure 1. Les questions que posent la qualité à l'agronome. D'après [5].

pour les filières courtes, et garantie d'une certaine stabilité de la qualité pour les acteurs de la filière (figure 1. Cet élargissement de son domaine d'étude s'accompagne de la prise en compte d'un niveau d'échelle supplémentaire : désormais, c'est le bassin d'approvisionnement des entreprises et des filières dans son ensemble qu'il convient d'appréhender, dans la mesure où il apparaît comme le territoire dans lequel se construit et s'améliore la qualité. La démarche de l'agronome, face à cette question de la qualité, se décline donc selon trois grands domaines d'action portant sur les problèmes de définition, de production, de garantie, chacun rapportés aux différents niveaux d'étude: de l'organe et la plante au bassin d'approvisionnement des entreprises et filières (figure 2). Les travaux impliqués s'étendent ainsi des questions de traduction en caractéristiques des plantes assez classiques jusqu'à l'élaboration des cahiers des charges de signes et de normes en collaboration avec les acteurs.

Cette présentation synoptique permet de dégager deux postures: une posture nordouest pour laquelle la qualité est un ensemble de caractéristiques et de critères pour lesquels il convient d'identifier les systèmes de culture 


\begin{tabular}{|c|c|c|c|}
\hline $\begin{array}{l}\text { Activité } \\
\text { Echelle }\end{array}$ & Définition & Production & Garantie \\
\hline Organe et plante & $\begin{array}{l}\text { Traduction en } \\
\text { caractéristiques } \\
\text { des plantes }\end{array}$ & $\begin{array}{c}\text { Fonction } \\
\text { nement des } \\
\text { plantes et sols }\end{array}$ & $\begin{array}{l}\text { Indicateurs } \\
\text { plante/sol }\end{array}$ \\
\hline $\begin{array}{c}\text { Parcelle et } \\
\text { peuplement végétal }\end{array}$ & $\begin{array}{l}\text { Hiérarchisation } \\
\text { critères visés à } \\
\text { la parcelle }\end{array}$ & & $\begin{array}{l}\text { Indicateurs suivi } \\
\text { des cultures }\end{array}$ \\
\hline $\begin{array}{l}\text { Parcelles et soles } \\
\text { des exploitations }\end{array}$ & $\begin{array}{l}\text { Déterminants } \\
\text { adoption cahiers } \\
\text { des charges }\end{array}$ & $\begin{array}{l}\text { Marges de } \\
\text { nanœurre des } \\
\text { agriculteurs }\end{array}$ & $\begin{array}{c}\text { Dispositifs de } \\
\text { certification des } \\
\text { EA }\end{array}$ \\
\hline $\begin{array}{l}\text { Bassin } \\
\text { d'approvisionnement } \\
\text { Entreprises et filières }\end{array}$ & $\begin{array}{l}\text { Objectifs issus } \\
\text { coordinations } \\
\text { agriculteurs + } \\
\text { utilisateurs }\end{array}$ & $\begin{array}{l}\text { Systèmes } \\
\text { techniques } \\
\text { complexes }\end{array}$ & $\begin{array}{c}\text { Elaboration des } \\
\text { CdC, signes et } \\
\text { normes }\end{array}$ \\
\hline
\end{tabular}

Figure 2. Les actions et les niveaux d'échelle. D'après [5].

permettant de les obtenir, et une seconde posture sud-est, pour laquelle la qualité résulte d'une combinaison d'organisations.

\section{Posture 1. La qualité comme compromis entre différentes caractéristiques des plantes}

La qualité se résume ici à des caractéristiques des organes récoltés, traduction des besoins des utilisateurs (consommateurs, industriels...). Pour atteindre les seuils voulus, il faut mettre en place des techniques culturales ad hoc à l'échelle des parcelles.

De ce point de vue, comment se présentent les questions de définition, de production, de garantie?

\section{C'est quoi un bon fruit ?}

Qu'est-ce qu'une bonne pêche? Cette question est difficile dans la mesure où elle met en évidence des antagonismes entre critères qu'il faut prendre en compte avec des critères biologiques difficiles à contourner... et liés aux cinétiques de croissance (figure 3) : I'accumulation des sucres est croissante dans le temps, contrairement à la fermeté du fruit. Obtenir à la fois un fruit relativement ferme et sucré, avec un bon rapport sucre sur acide, devient difficile à préciser même biologiquement.

Le melon (figure 4) doit être d'un bon calibre, sa chair doit être sucrée et il ne doit pas être vitrescent (il ne doit pas y avoir à l'intérieur des poches d'eau). Ici également des compromis doivent être passés sur la définition quand on bâtit un schéma d'élaboration de la qualité en s'appuyant sur les critères écophysiologiques pour remonter vers des critères de milieu et des systèmes de culture qui peuvent permettre de le modifier. Dans cette situation, il convient de jouer sur l'humidité, la température, la charge en fruit, la pollinisation et la date de récolte. Par souci d'efficacité, les acteurs de l'interprofession proposent pour différents fruits un indicateur cumulé, optimisant la combinaison de ces différents paramètres. Ils s'efforcent de corréler avec le goût des consommateurs. L'agronome s'appuie sur une démarche d'analyse des différents composants de la qualité, afin de trouver un compromis au niveau des systèmes de culture.

\section{Comment garantir la qualité d'un bon fruit?}

Nous sommes toujours ici à cette échelle du travail sur le fonctionnement des plantes (figure 5).

Pour être appréciées du consommateur, les bananes doivent être moelleuses et sucrées, mais elles doivent aussi être transportables sans dommage depuis les lieux de production. Entre le moment où elle est récoltée et le moment où elle est mûre, le potentiel de conservation traduit en « durée vie verte » (DVV) est extrêmement variable. Si la DVV est trop longue, la banane arrive sur la table du consommateur verte avec plutôt des amidons et peu de sucres, mais elle a été transportée dans des conditions parfaites à la satisfaction de l'exportateur et du grossiste. Si la DVV est trop courte la banane sera plus grosse et plus riche en sucre mais... «écrasée ». On a donc tenté de créer au moyen d'observations écophysiologiques, un indicateur d'aide à la décision pour choisir la date de récolte réalisant un compromis entre les contraintes du transport et les exigences de la consommation. Cet indicateur mesure en

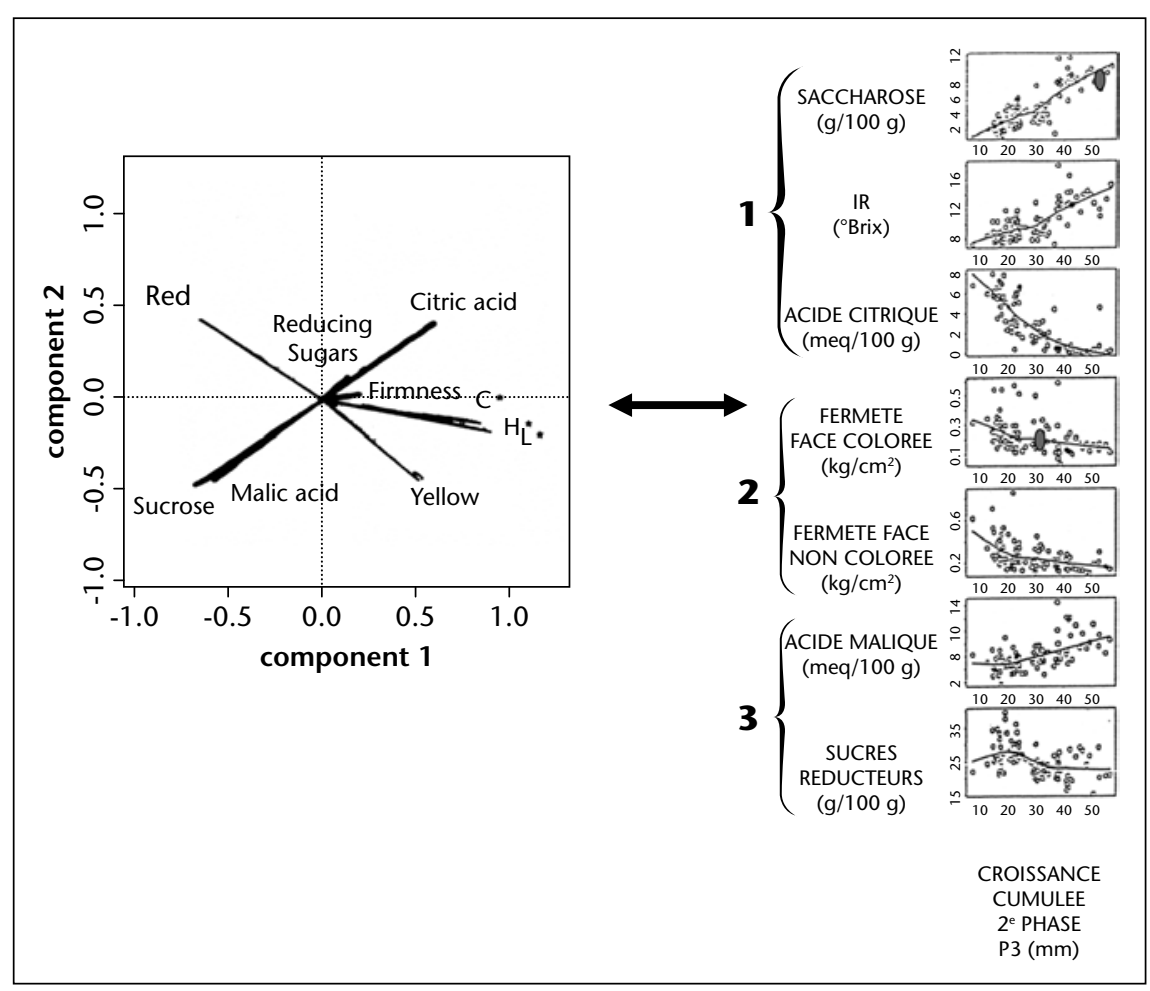

Figure 3. C'est quoi un bon fruit? Dans le cas de la pêche, la qualité renvoie à des antagonismes entre critères qu'il faut prendre en compte... très liés aux cinétiques de croissance. D'après [3]. 


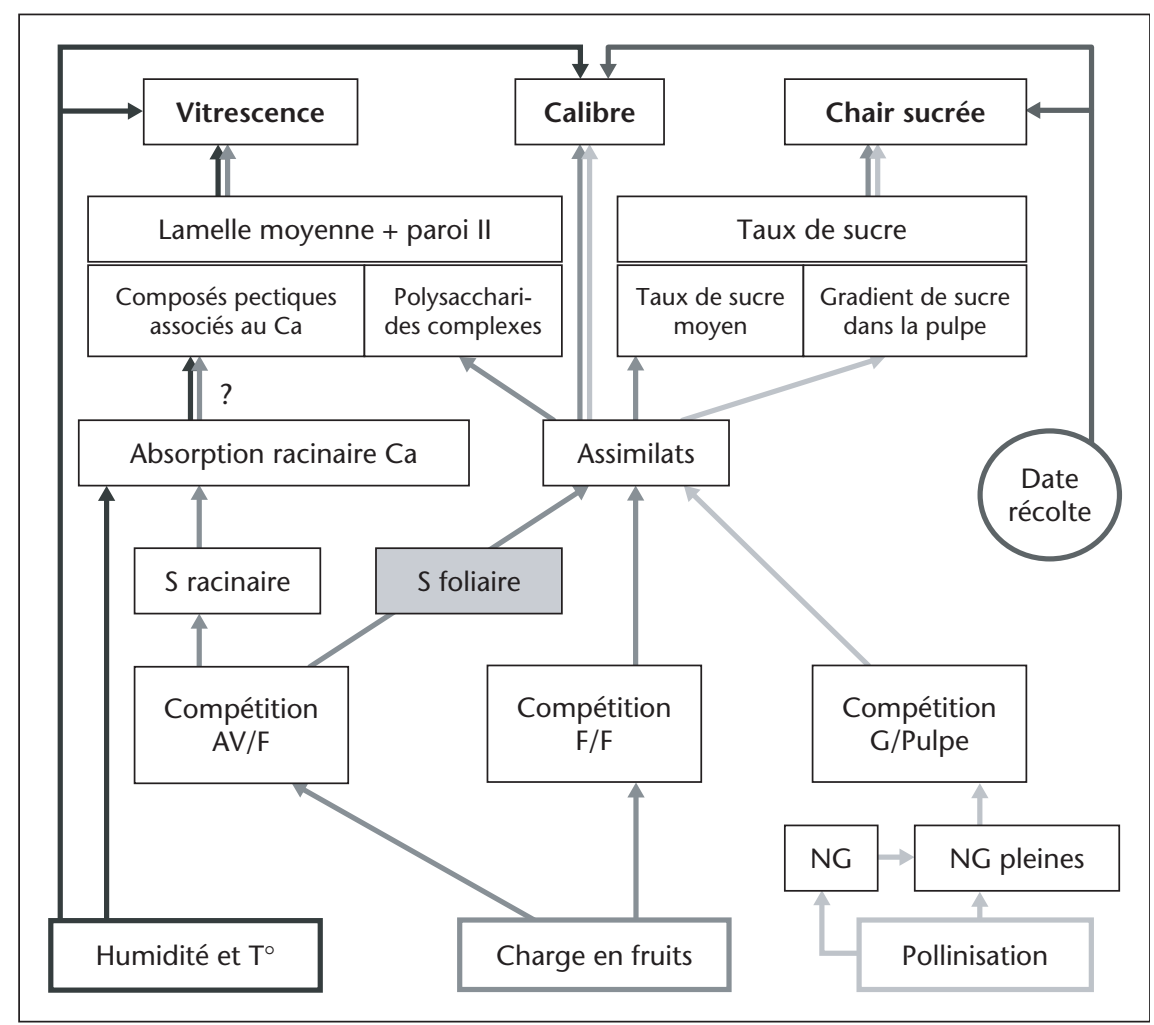

Figure 4. Comment cela se fabrique un bon fruit?

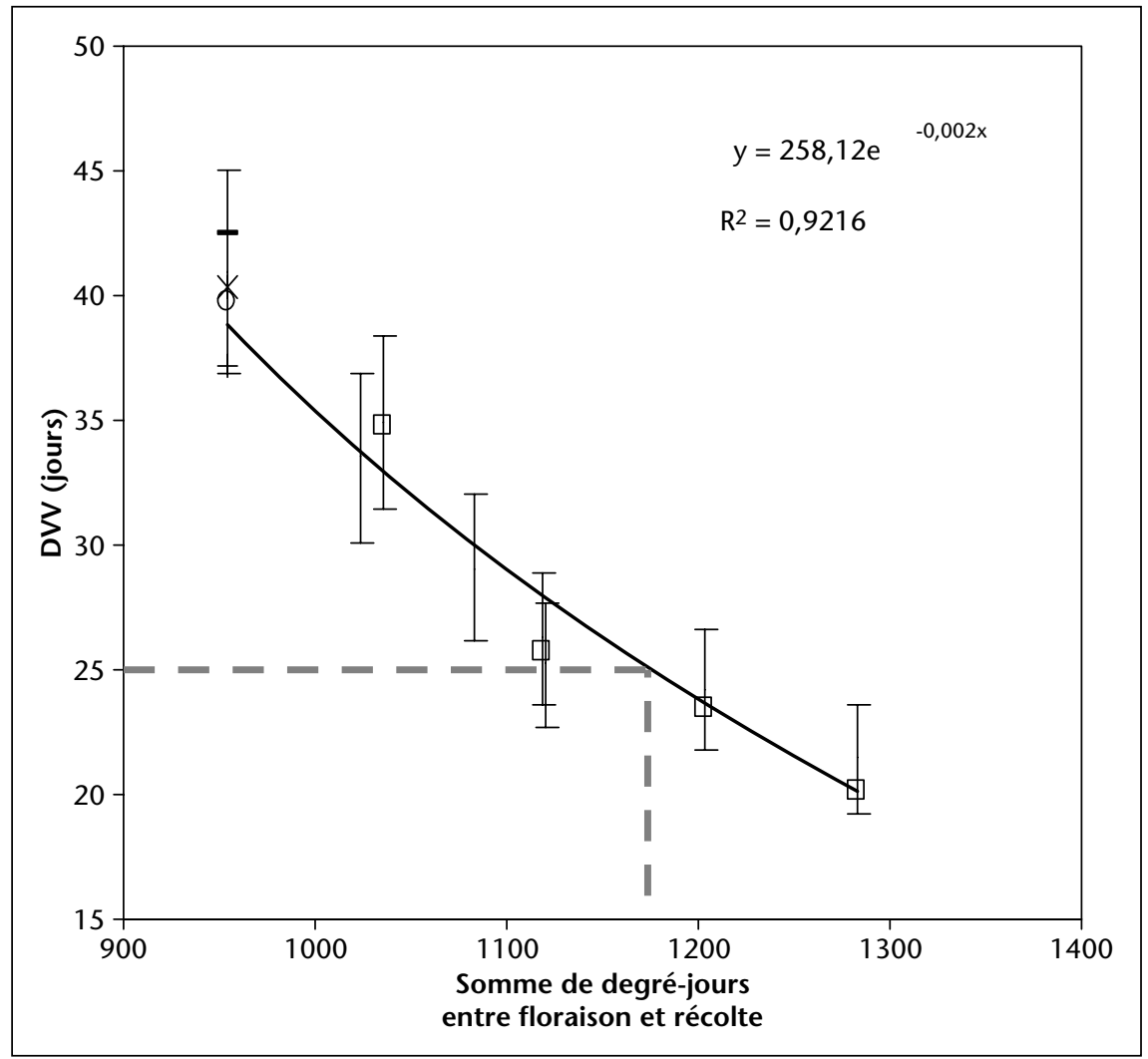

Figure 5. Comment garantir la qualité d'un bon fruit ? Pour la banane une date de récolte à 1200 degrés-jours après le stade "floraison", garantit un poids suffisant et une durée de vie verte (25 jours) ni trop courte ni trop longue. D'après $[1,4]$.
« degré-jour » la durée de la période séparant la floraison de la récolte. Pour une durée de vie verte objectif de 25 jours, qui correspond à peu près à la durée de transfert de la banane par bateau vers l'Europe, une date de récolte à 1200 degrés-jours après le stade « floraison » garantit ainsi un poids et une maturité suffisants.

\section{Posture 2. La qualité comme compromis entre différentes organisations}

La définition de la qualité est l'objet de vives discussions dans la mesure où elle porte sur des critères évolutifs et donc négociables et sur les moyens d'atteindre ces critères. À l'échelle de la production, les performances des systèmes sont variables, dans le temps, dans l'espace, entre exploitations, et la question se pose de savoir comment gérer cette variabilité.

Cette variabilité peut être réduite ou valorisée par la mise en place de dispositifs de qualification des produits.

\section{L'adaptation a une variabilité dans le temps}

L'évolution au cours de l'année de la teneur en nitrates des laitues, d'après des prélèvements effectués dans un certain nombre de magasins en Suisse, révèle des taux plus élevés en décembre et janvier, qu'au cœur de l'été (figure 6). Or, on sait qu'un taux élevé de nitrates peut induire des risques pour la santé mais que le nitrate est un osmoticum ayant pour effet de stimuler les échanges en eau : ce qui laisse le choix entre une salade pauvre en nitrate et fripée et une salade feuillue, bien croquante et riche en nitrate, surtout en hiver.

En 1991 a été mise en place une norme européenne fixant un seuil maximal de nitrates dans les salades tenant compte de manière explicite de cette variabilité temporelle (la ligne brisée sur le schéma). Ainsi, après négociation, la réglementation a été adaptée à un fait biologique difficile à contourner dans des conditions économiques raisonnables (on pourrait faire les salades sous serres avec de la lumière). À une date donnée, y compris en hiver, il est possible de réduire la teneur en nitrate pour s'adapter à la norme en faisant évoluer les systèmes de cultures [2].

\section{La gestion de la variabilité dans l'espace}

L'orge destiné à faire de la bière doit satisfaire une vingtaine de critères technologiques, comme la viscosité, la friabilité, etc., tous liés à la technologie du process même du brasseur (figure 7). 


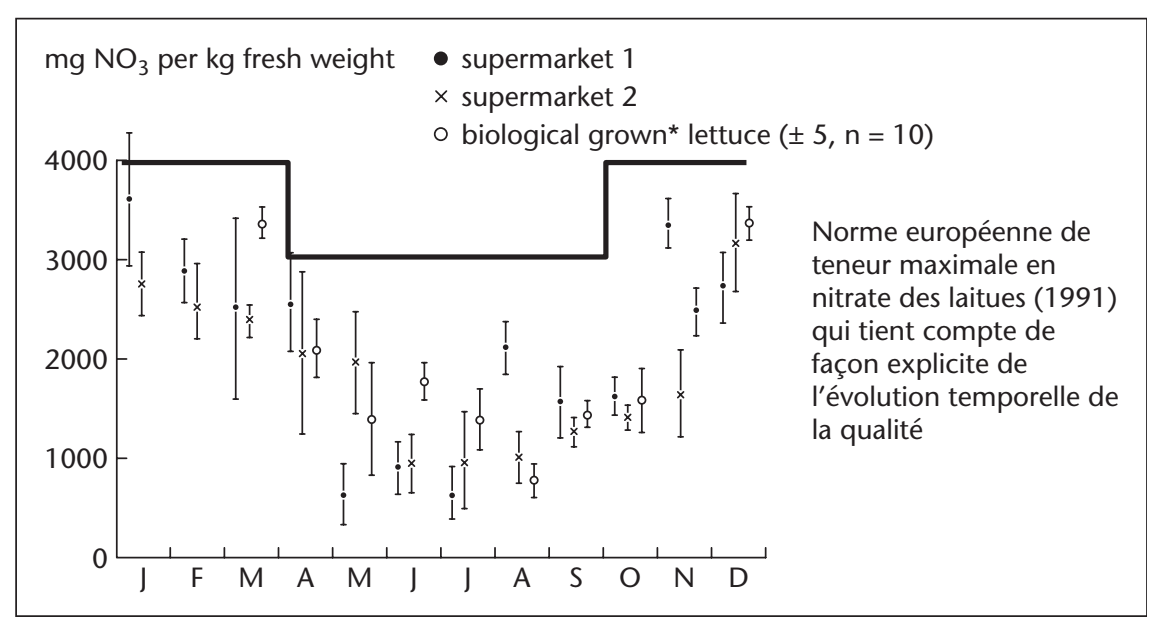

Figure 6. Définition de la qualité : les composantes temporelles. Exemple : la teneur en nitrate des laitues, un critère de qualité sanitaire qui évolue au cours du temps. D'après Temperli et al., 1978-79, cité dans [2].

Au niveau du malteur ce nombre diminue et au niveau de la parcelle les critères ne portent plus que sur le taux de protéine (inférieur à 11,5\%) le calibre supérieur à $2,5 \mathrm{~mm}$ et le bon taux de germination ceci avec quelques variétés conseillées. Cette simplification a plusieurs raisons. Une viscosité, une friabilité, etc. sont difficiles et chers à mesurer à la parcelle, mais seraient-ils mesurés, l'établissement de relations avec des états du milieu et des systèmes de culture s'avèrent particulièrement comple$x e s$. Si bien qu'aux traits horizontaux marquant ce que recherche le brasseur en matière de viscosité, correspondent les deux traits verticaux délimitant ce qui est demandé à l'agriculteur en matière de taux protéines. La « réduction» de la qualité au critère «taux de protéines » est un compromis entre les acteur$s$ dans lequel brasseur et malteur prennent un risque vis-à-vis de leurs besoins (la corrélation taux de protéines-viscosité est faible) mais qui permet aux agriculteurs et aux coopératives d'identifier un levier d'action, imparfait mais mesurable, pour améliorer leur réponse au marché.

Ce levier d'action peut être mobilisé à l'échelle de la parcelle ou à une échelle supérieure, sous la forme d'un travail post-récolte consistant à mélanger ou à trier les graines de différentes origines.

Classiquement, les agronomes jouent sur les modifications des systèmes de culture afin de resserrer la variabilité des critères déterminants la qualité. Dans certains contextes de marché, il

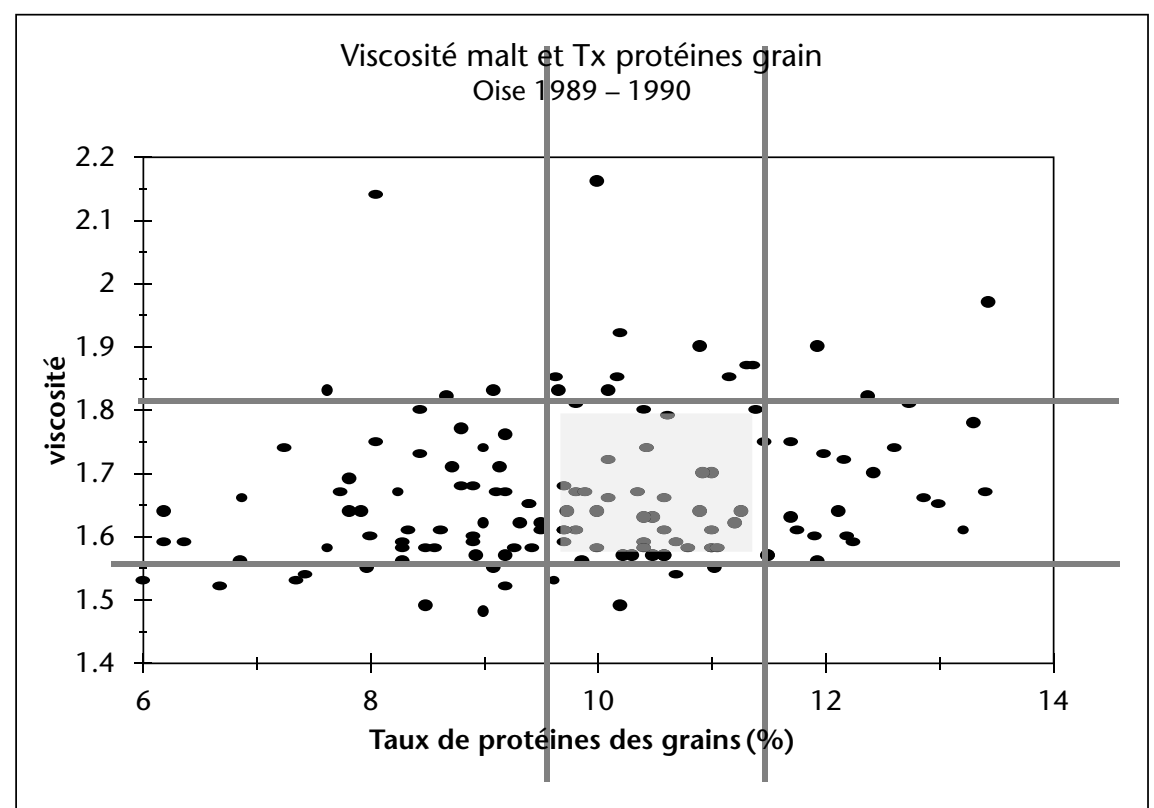

Figure 7. Définition de la qualité : négociation entre acteurs : exemple de l'orge [5].

convient moins de modifier les systèmes de culture que de trier les parcelles, de repérer dans le bassin d'approvisionnement ce qui serait bon pour faire du blé tendre pour le pain, ou du blé tendre pour les biscuits, sans toucher au système de culture, sans modifier l'organisation des exploitations agricoles. La question de la coexistence entre OGM et non-OGM est de cet ordre : on peut jouer sur les systèmes de culture, mais on peut aussi intervenir sur la manière de trier les parcelles dans un territoire. On peut également trier en se fondant sur I'origine : cela s'applique pour le vin (les AOC) [8] mais peut s'appliquer également aux commodités comme la canne destinée au sucre ou le blé destiné au pain. La question est dans ce cas de caractériser globalement, sans rentrer dans les détails des différents critères, ces caractéristiques d'origine.

Pour en revenir à l'orge de brasserie, les itinéraires techniques proposés visaient à obtenir des rendements importants avec des taux de protéines faibles. On s'aperçoit (figure 8) que $90 \%$ des itinéraires techniques conseillés (en clair) apportent bien les résultats attendus. À l'opposé $39 \%$ des itinéraires « non-conseillés » (en sombre) conduisent à des taux de protéines supérieurs à $11,5 \%$. Si l'agriculteur n'a pas nécessairement intérêt à choisir l'itinéraire proposé - itinéraires qui exigent plus d'observations, de calculs - il n'en va pas de même pour le collecteur. Ces données deviennent donc des outils de négociation entre agriculteurs et opérateurs. Là il y a quelque chose de plus complexe que l'approche système de culture/critère.

Dans les questions de tri on utilise des modèles de simulation du rendement et des protéines sur un territoire donné de manière précoce, afin de classer les parcelles d'un bassin dans l'ordre croissant des valeurs simulées (figure 9). Là encore l'agronome se positionne au-delà de la parcelle et du système de culture, voire au-delà de l'exploitation afin de trier 4000 t à $11,6 \%$ de taux de protéine et $4000 \mathrm{t}$ à $12,5 \%$ de taux de protéine éventuellement destinés à des marchés différents. On a là un niveau déterminant de la qualité et d'organisation de la qualité très différent des niveaux abordés classiquement.

Pour mémoire, sur la qualité d'origine, ce travail sur les terroirs des Côtes-du-Rhône méridionales (figure 10). Les travaux ont porté sur la définition d'unité de terroir qui explique à travers les composantes géomorphologiques les effets sur la qualité des vins.

Mais la mise au point d'outils de pilotage de la qualité n'est pas toujours résolue, en particulier face à des événements rares au cours desquels la qualité est brutalement dégradée.

Les mycotoxines en céréales (toxines provoquées par l'activité de champignons - Fusarium 


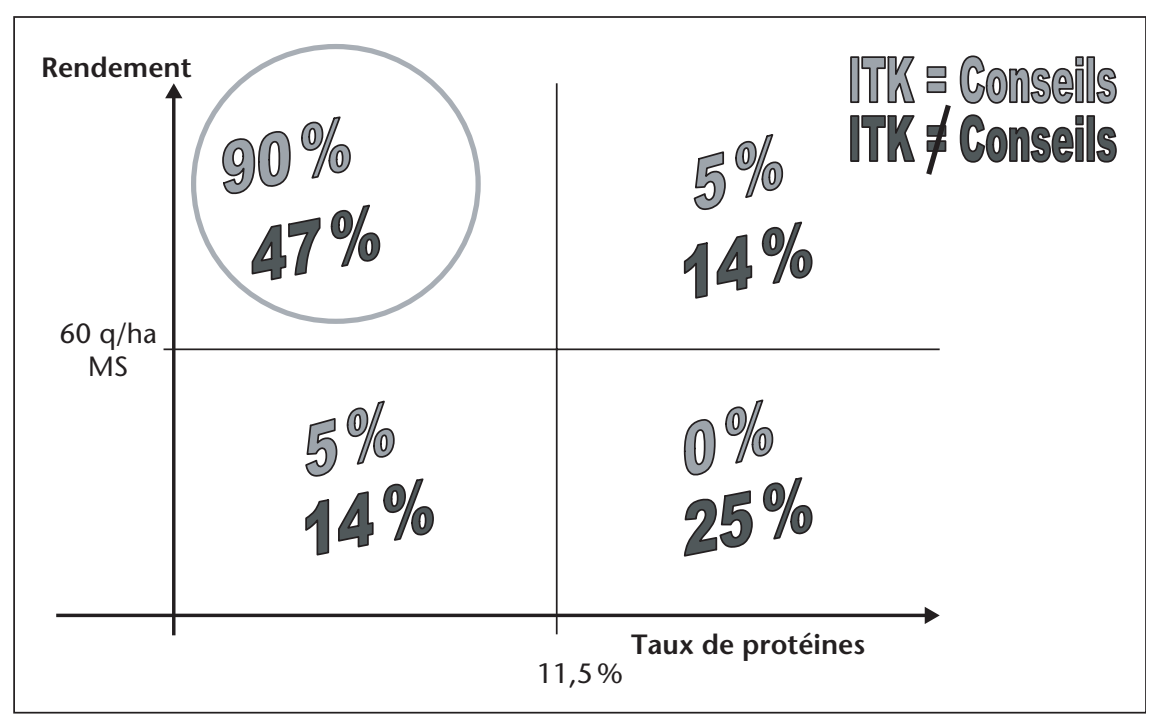

Figure 8. Evaluation des itinéraires techniques sur orge de brasserie. D'après [6].

en particulier - développés sur les épis), en tout les cas en blé, sont relativement rares mais peuvent être très élevées dans quelques parcelles. Qui plus est les connaissances sont ici incomplètes : les mécanismes fins de l'accumulation des mycotoxines sont encore loin d'être établis.
Et pourtant le règlement est tombé : il faut être à moins de $1250 \mu \mathrm{g} / \mathrm{kg}$ de mycotoxine.

Une grille des pratiques à risque a donc été établie par Arvalis fondée sur l'analyse statistique de bases de données, qui précise par exemple que lorsqu'on ne travaille pas le sol après un précédent maïs, les risques sont

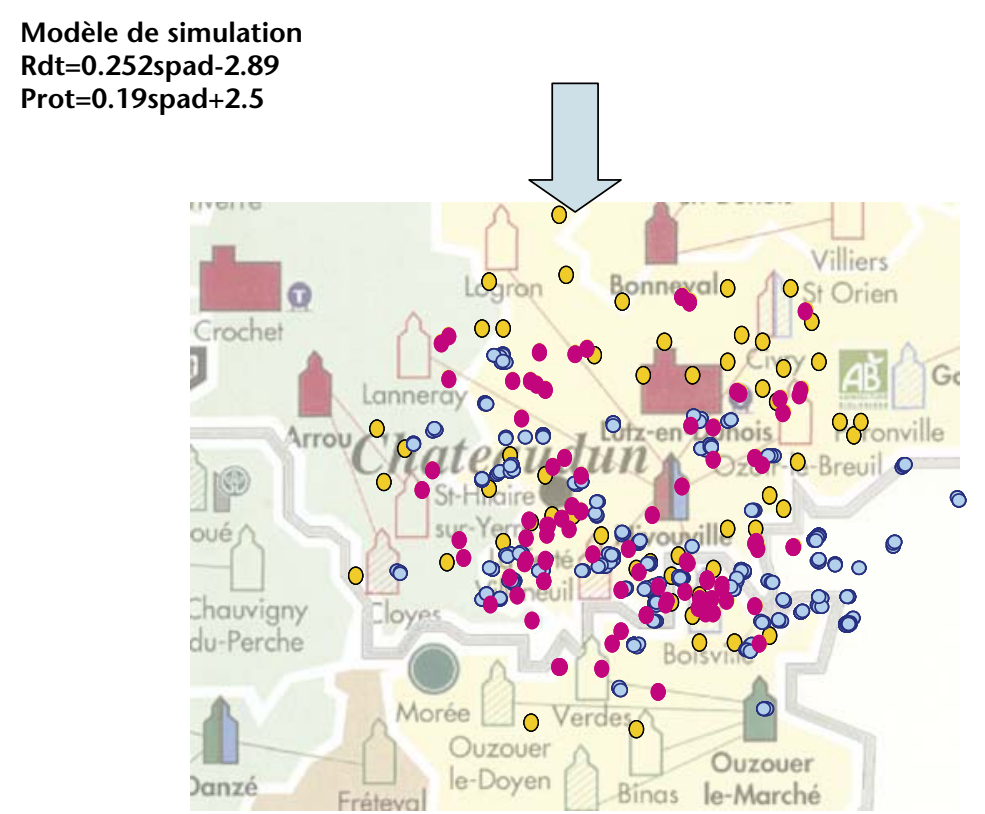

Classement des parcelles du bassin dans I'ordre croissant des valeurs

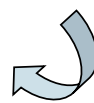
simulées

\section{$4000 \mathrm{t}$ à $11,6 \% \mathrm{tx}$ prot}

$4000 \mathrm{t}$ à $12,5 \%$ tx prot importants. Vont ainsi être limités les précédents maïs pour un blé sans labour. Mais le labour, par ailleurs, est réduit dans certaines exploitations pour des raisons de travail, d'énergie, d'évolution de la structure et de la microfaune du sol...

Une autre solution consisterait aussi à repérer les situations à risque et à y faire des mesures avant récolte pour vérifier la réalité du problème car il y a une corrélation entre la déoxynivalénol présente dans le blé et celle qui sera présente le jour de la récolte.

Les mycotoxines viennent du champ, mais elles sont aussi dans les stocks, ce qui oblige également à considérer cet autre aspect du problème et si la dégradation de la qualité au cours du stockage est peu présente sous nos latitudes, on sait qu'elle peut être tragique dans un certain nombre de pays.

\section{Les questions de garantie}

Les différents types d'action ainsi que les modes d'organisation évoqués précédemment, sont aujourd'hui couverts par un certain nombre de signes (figure 11): des signes qui remontent vers le consommateur et des signes liés à la production comme l'agriculture raisonnée, Quali'Terre, Agri confiance et qui sont repris par les industriels et les distributeurs pour les amener vers le consommateur.

\section{Conclusion}

La qualité des productions végétales relève de l'amélioration des systèmes de culture à la parcelle mais aussi des organisations entre acteurs. Elle implique :

- une définition multiforme, négociée et en constant changement ;

- une production qui combine différentes marges de manœuvre de l'échelle de la parcelle à l'assiette du consommateur ;

- des garanties qui s'appuient sur des indicateurs techniques et organisationnels pour réduire les incertitudes.

Mais de nouvelles questions et tendances apparaissent.

La multiplication des signes valant pour garantie de la qualité des produits et des manières de produire n'améliore pas la visibilité de la qualité. Est-ce la raison pour laquelle on assiste aujourd'hui à un déplacement des consommateurs vers les circuits courts?

À ce niveau que devient la qualité ? La qualité devient "manger son territoire», valoriser la diversité, réduire les coûts énergétiques, soit un ensemble de caractéristiques qui devront à l'avenir êtres abordées de plain-pied et qui montrent que la qualité ira bien au-delà des aspects nutritionnels.

Nous dirigeons-nous vers la mise en place d'une qualité durable, intégrant les dimensions 


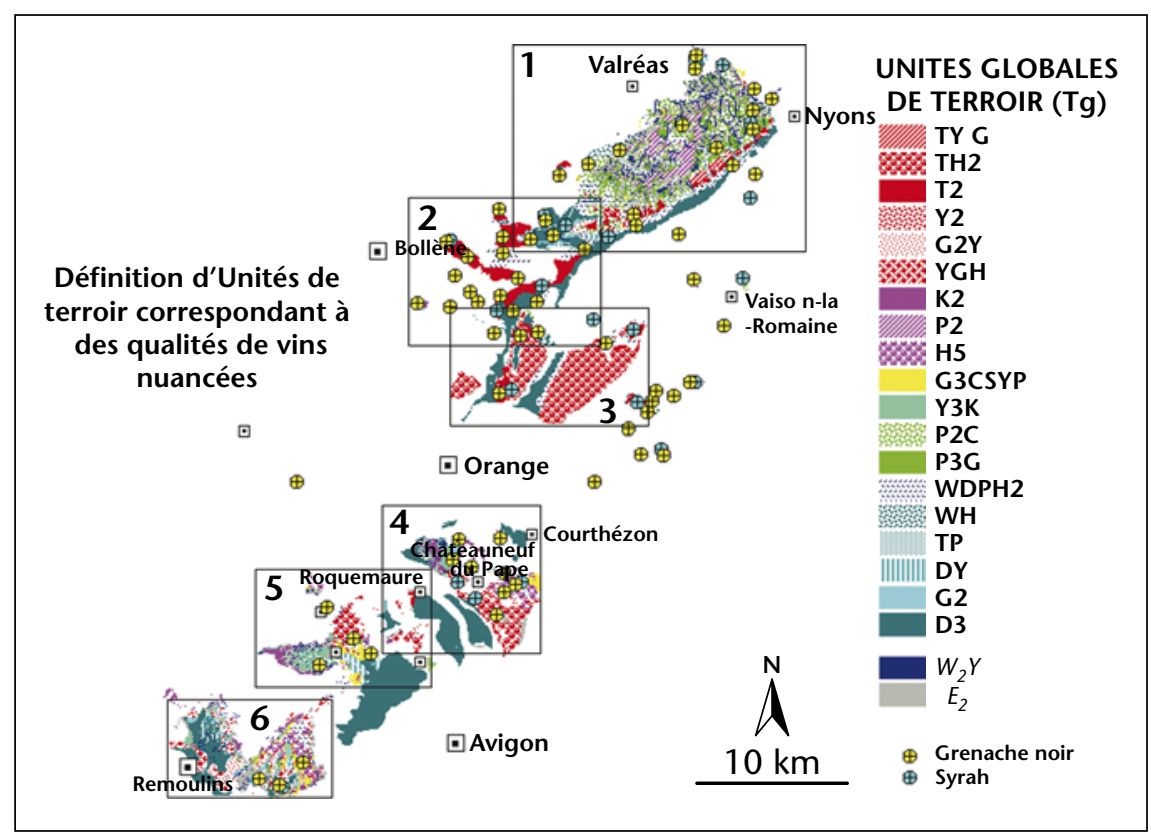

Figure 10. Terroirs en Côtes-du-Rhône méridionales. D'après [8].

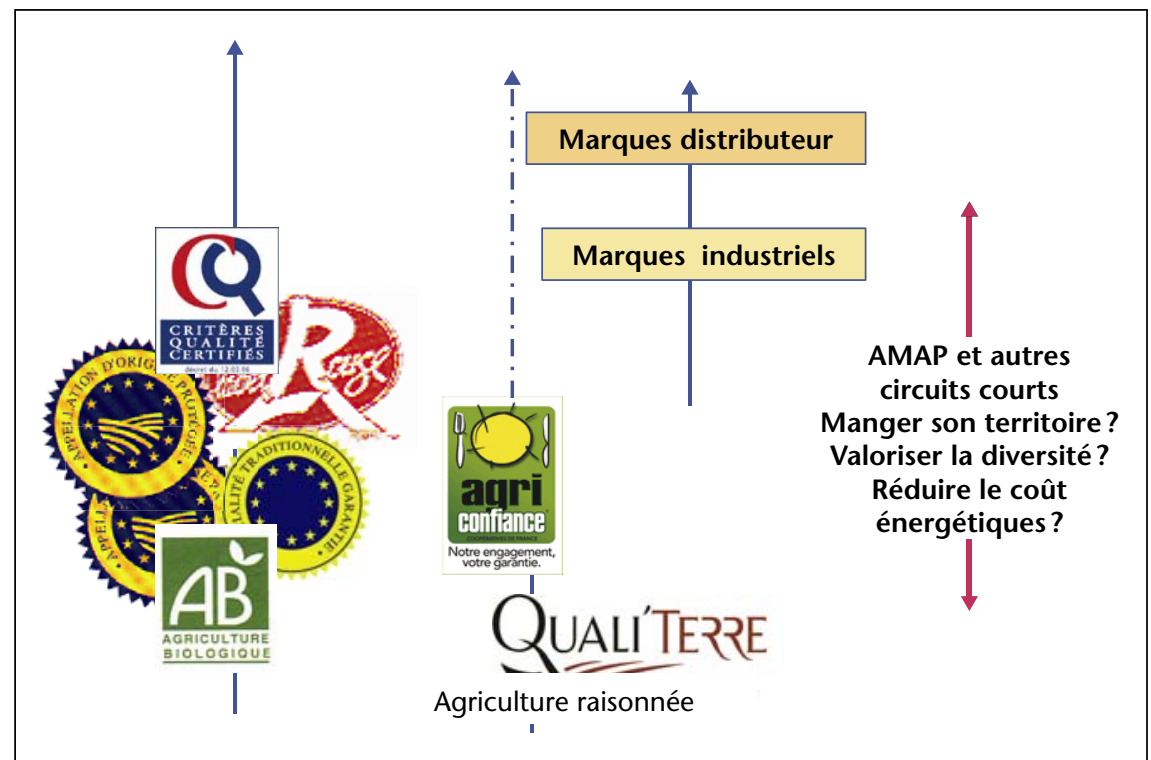

Figure 11. Une multiplication de signes pour garantir la qualité des produits et les manières de produire. environnementales, les bilans énergétiques, les aspects de proximité ? Cette intégration est urgente pour conforter les consommateurs. On ne peut pas se satisfaire de réflexions entendues récemment dans un colloque sur les circuits courts affirmant que "parce qu'on connaissait le producteur le produit était bon ».

\section{RÉFÉRENCES}

1. BUGAUD C, LASSOUDIERE A. Variabilité de la durée de vie verte des bananes en conditions réelles de production. Fruits $2005 ; 60: 227$ 36.

2. DAPOIGNY L, DE TOURDONNET S, ROGERESTRADE I, JEUFFROY MH, FLEURY A. Effect of nitrogen nutrition on growth and nitrate accumulation in lettuce (Lactuca sativa L.), under various conditions of radiation and temperature. Agronomie 2000 ; 20(2000) : 843-55.

3. GENARD M, BRUCHOU C, SOUTY M. Variabilité de la croissance et de la qualité chez la pêche (prunus persica $L$ Batsch) et liaison entre croissance et qualité. Agronomie 1991; 11 : 829-945.

4. jullien A, CHILlet M, maléZieuX e. Preharvest growth and development, measured as accumulated degree days, determine the postharvest green life of banana fruit. I Hortic Sci Biotechnol (in Press).

5. LE BAIL M. Contribution aux recherches agronomiques sur la qualité des productions végétales: du système de culture au système local d'approvisionnement. Hdr, INPL, Nancy, 2005.

6. LE BAIL M, MEYNARD JM. Yield and protein concentration of spring malting barley: the effects of cropping systems in the Paris Basin (France). Agronomie $2003 ; 23$ : 13-27.

7. LE BAIL M, JEUFFROY MH, BOUCHARD C, BARBOTTIN A. IS it possible to forecast the grain quality and yield of different varieties of winter wheat from Minolta SPAD meter measurements? Eur J Agron 2005 ; 23 : 379-91.

8. VAUDOUR E. Les terroirs viticoles. Paris : Dunod, 2003 ; (294 pp.). 